\title{
PDK2-enhanced glycolysis promotes fibroblast proliferation in thyroid-associated ophthalmopathy
}

\author{
Ruiqi Ma1,2,*, Lu Gan1,2,*, Hui Ren11, Andrew Harrison³ and Jiang Qian1 \\ 'Department of Ophthalmology, Fudan Eye \& ENT Hospital, Shanghai, China \\ 2Laboratory of Myopia, Chinese Academy of Medical Sciences, Shanghai, China \\ 3Department of Ophthalmology and Visual Neurosciences, University of Minnesota, Minneapolis, Minnesota, USA
}

Correspondence should be addressed to A Harrison or J Qian: harri060@umn.edu or qianjiang@fudan.edu.cn

*(R Ma and L Gan contributed equally to this work)

\begin{abstract}
The study aimed to investigate the role of pyruvate dehydrogenase kinase (PDK) in regulating glycolysis and proliferation of perimysial orbital fibroblasts (pOFs) during the pathogenesis of thyroid-associated ophthalmopathy (TAO). EdU and BrdU incorporation assays were performed to examine cell proliferation. Lactate production and oxygen consumption assays were conducted to evaluate glycolysis. Real-time PCR was adapted to quantify PDK mRNA levels. Capillary Western immunoassay was adapted to quantify PDK2, Akt, pAkt 308 and GAPDH in protein samples. The TAO pOFs exhibited stronger proliferation activity, higher intracellular lactate concentration, and lower oxygen consumption rate than the control pOFs. The PDK inhibitor dichloroacetic acid (DCA) dose-dependently suppressed the proliferation of both TAO and control pOFs. DCA reduced lactate production and promoted oxygen consumption in the TAO pOFs but showed no significant effects on glycolysis in the control pOFs. Among four PDK isotypes, PDK2 was overexpressed in the TAO pOFs. The potential PDK signaling mediator, cytoplasmic Akt, was more abundant in TAO pOFs than control pOFs. Knockdown of PDK2 resulted in lower lactate production, stronger oxygen consumption, weaker proliferation activity, and less cytoplasmic Akt in the TAO pOFs but showed no significant effects in the control pOFs. The Akt inhibitor MK2206 suppressed proliferation in both TAO and control pOFs, and lactate production was inhibited by MK2206 in the TAO OFs but not the control pOFs. To conclude, PDK2 overexpression enhances glycolysis and promotes proliferation via Akt signaling in the TAO pOFs. These findings yield insights that PDK2 is a potential therapeutic target for TAO treatment.
\end{abstract} Key Words

\section{Introduction}

Thyroid-associated ophthalmopathy (TAO), commonly co-occurrence with thyroid dysfunction (mostly Graves' disease), is an autoimmune disorder of orbital tissues. This condition is disfiguring and potentially sight-threatening, because its pathological changes culminate in the expansion and remodeling of the soft tissues within the orbit. Only a few medical therapies have been proven safe and effective to alter the clinical outcome of TAO. 
For instance, teprotumumab was recently approved by US FDA for the treatment of active, moderate to severe TAO (Smith 2019, Douglas et al. 2020). To develop more targeted treatments requires a more complete understanding of the mechanisms involved in the pathogenesis. Considerable effort has been expended in identifying the role of oxidative stress within the TAO orbit. Cigarette smoking, a putative cause of oxidative stress, is commonly blamed as a risk factor for developing TAO (Wiersinga et al. 2018). Reduction in tobacco use and supplement of selenium (an antioxidant agent) are frequently cited as effective strategies to reduce the incidence and morbidity of TAO (Taylor et al. 2020). Evidence from fundamental research suggests increased oxidative stress and upregulated antioxidant capacity in the serum and orbit of TAO patients (Korkmaz et al. 2015, Marique et al. 2015, Aass et al. 2016, Yuksel et al. 2020). Take together, these findings yield important insights into the role of oxygen metabolism during the pathogenesis of TAO.

The research findings thus far have established that orbital fibroblasts (OFs) represent the central target for immune reactivity in TAO development (Smith \& Janssen 2019). Many studies have examined the behavior of OFs with regard to their responses to antioxidants. Significant differences between the TAO and control OFs have been identified in aspects of proliferation, cytokine production, reactive oxygen species (ROS) generation and so forth (Byun et al. 2016, Choi et al. 2018, Ko et al. 2018, Lee et al. 2019). Details concerning the oxygen metabolism and its regulatory factors have not been fully investigated. In mammalian cells, there are two basic pathways to generate energy, glycolysis in the cytoplasm (requires no oxygen) and oxidative phosphorylation in mitochondria (requires oxygen). The pyruvate dehydrogenase kinase (PDK), which has four isoforms (PDK1-4), is considered as the gatekeeper enzyme to trigger the switch from oxidative phosphorylation to cytoplasmic glycolysis (Stacpoole 2017). Growing evidence supports the concept that the glycolytic pathway dominates in proliferating cells (especially tumor cells) which present with reduced oxygen consumption and increased lactate production (Vander Heiden et al. 2009, Martinez-Outschoorn et al. 2017).

Perimysial orbital fibroblast (pOF) is a unique phenotype of OFs that resides within extraocular muscle fibers. Our previous study implied that the TAO pOFs possessed stronger proliferative capacity than the control pOFs obtained from healthy subjects (Ma et al. 2018). Based on these observations, we hypothesized that glycolytic pathway may be preferentially enhanced in the TAO pOFs. To test the hypothesis, this study compared the differences between the TAO and control pOFs regarding proliferation, lactate production, oxygen consumption, PDK expression, cellular responses to PDK inhibition, and the mediator of PDK signaling transduction.

\section{Materials and methods}

\section{Materials}

Reagents included the PDK inhibitor dichloroacetic acid (DCA, Sigma-Aldrich, Cat\# D54702) and the Akt inhibitor MK2206 (Selleck, Cat\# S1078). Commercial kits included the Cell Counting Kit-8 (CCK-8, DOJINDO, Cat\# CK04), EdU incorporation assay (Life Technologies, Cat\# C10637), BrdU incorporation assay (Roche, Cat\# 11647229001), Lactate ELISA kit (Abcam, Cat\# ab65331), Deproteinizing Sample Preparation kit (Abcam, Cat\# ab204708), Oxygen Consumption Assay (Abcam, Cat\# ab197243), rtPCR kit (TaKaRa, Cat\# RR047), SYBR qPCR kit (TaKaRa, Cat\# RR420), Lipofectamine RNAiMAX Reagent (Invitrogen, Cat\# 13778), Protein and RNA Isolation System (PARIS, Life Technologies, Cat\# AM1921), and Capillary Western Immunoassay kit (ProteinSimple, 12-230 kDa). Primary antibodies targeted vimentin (clone GT7812; Abcam, Cat\# ab184631), PDK2 (clone EPR1948Y; Abcam, Cat\# ab68164), Akt (clone 11E7; CST, Cat\# 4685), pAkt ${ }^{308}$ (clone L32A4; CST, Cat\# 5106), and GAPDH (clone 14C10; CST, Cat\# 2118). Validations of the primary antibodies are provided on the manufacturer's websites.

\section{Subject recruitment}

Extraocular muscle tissues were collected from six TAO patients during orbital decompression surgery and from six control volunteers during orbital or strabismus surgery (Supplementary Table 1, see section on supplementary materials given at the end of this article). The TAO subjects were patients at inactive stage with clinical activity score less than 3 points on a 7-point scale consisting of eyelid swelling, eyelid erythema, conjunctival redness, chemosis, caruncle or plical inflammation, spontaneous orbital pain, and gaze evoked orbital pain. The control subjects were volunteers without thyroid disease or other autoimmune disease. The exclusion criteria were diabetes or impaired glucose tolerance, thyroid dysfunction, orbital infection or orbital fracture within 6 months, and previous intraorbital malignant tumors. Written informed consent was obtained from each subject, and the study protocol was approved by the Institutional Review Board of Fudan Eye \& ENT Hospital. 


\section{Cell culture}

Primary pOFs were isolated from extraocular muscle tissues as previously described (Ma et al. 2018, 2019). After reaching $90 \%$ confluence, the primary pOFs were passaged with $0.25 \%$ trypsin and maintained in high-glucose DMEM (Gibco, 11965) supplemented with fetal bovine serum (FBS, $10 \%, \mathrm{v} / \mathrm{v})$, penicillin (100 IU/mL) and streptomycin (100 $\mu \mathrm{g} / \mathrm{mL}$ ). Passages 3-8 were used in the study.

\section{Cell viability}

The CCK-8 colorimetric assay was adapted to measure cell viability. Passaged pOFs were seeded in 96-well plates at a density of 8000 cells/well and incubated overnight for recovery. After starvation for $6 \mathrm{~h}$, the pOFs were incubated with different concentrations of DCA (0-150 mM) in $1 \%(\mathrm{v} / \mathrm{v})$ FBS for $30 \mathrm{~min}$, treated with CCK-8 reagent $(10 \%, \mathrm{v} / \mathrm{v})$ for $25-45 \mathrm{~min}$, and processed for absorbance measurement at $450 \mathrm{~nm}$.

\section{EdU incorporation assay}

The EdU incorporation assay is an immunofluorescence method to semi-quantify cell proliferation. Passaged pOFs were seeded on glass coverslips in 24-well plates and incubated for $24 \mathrm{~h}$ for recovery. The pOFs were starved for $12 \mathrm{~h}$, treated with DCA (0-150 mM) in 1\% (v/v) FBS for $48 \mathrm{~h}$, and incubated with EdU labeling solution $(10 \mu \mathrm{M})$ during the last $24 \mathrm{~h}$. The incorporated EdU was detected according to the manufacturer's protocol, and the cellular nuclei were labeled with DAPI $(1: 1000)$ staining. A Leica TCS SP2 confocal microscope was used for imaging.

\section{BrdU incorporation assay}

The BrdU incorporation assay is an ELISA method to quantify cell proliferation. Passaged pOFs were seeded in 96-well plates and incubated overnight for recovery. The pOFs were starved for $12 \mathrm{~h}$, treated with DCA (0-150 mM) in $1 \%(\mathrm{v} / \mathrm{v})$ FBS for $48 \mathrm{~h}$, and incubated with BrdU labeling solution $(10 \mu \mathrm{M})$ during the last $12 \mathrm{~h}$. The incorporated BrdU was conjugated with anti-BrdU antibody for $90 \mathrm{~min}$, and the substrate solution was added for 5-10 min for color development. The absorbance values were measured at $370 \mathrm{~nm}$ with a reference wavelength at $492 \mathrm{~nm}$.

\section{Lactate production assay}

Passaged pOFs were seeded in $15 \mathrm{~cm}$ dishes to reach $90 \%$ confluence, deprived of serum for $6 \mathrm{~h}$, incubated with
DCA (0-15 mM) or MK2206 (0-3 $\mu \mathrm{M})$ in 1\% (v/v) FBS for $12 \mathrm{~h}$, and harvested for intracellular lactate measurement according to the instruction of Lactate ELISA kit. In brief, the cell pellet was homogenized in $200 \mu \mathrm{L}$ Lactate Assay Buffer. After centrifugation, the supernatant was collected and processed with the Deproteinizing Sample Preparation kit to remove endogenous lactate dehydrogenase. The lactate concentration in each sample was determined against the standard curve.

\section{Oxygen consumption assay}

Passaged pOFs were seeded in 96-well plates at a density of $4 \times 10^{4}$ cells/well and incubated overnight for recovery. The pOFs were starved for $6 \mathrm{~h}$ and treated with DCA (0-15 $\mathrm{mM})$ or MK2206 $(0-3 \mu \mathrm{M})$ in $1 \%(\mathrm{v} / \mathrm{v})$ FBS for $12 \mathrm{~h}$. All wells were then replaced with $150 \mu \mathrm{L}$ fresh culture medium containing $10 \mu \mathrm{L}$ Extracellular Oxygen Consumption Reagent and promptly sealed by pre-warmed High Sensitivity Mineral Oil. Measurement was repeated at 0.5 min intervals for $200 \mathrm{~min}$ at $380 \mathrm{~nm}$ excitation and 650 $\mathrm{nm}$ emission wavelengths. The oxygen consumption rate was calculated based on the slope of the ascending linear proportion of the signal profile.

\section{Immunofluorescence staining}

Passaged pOFs were grown on glass coverslips for immunofluorescence staining. The pOFs were fixed with paraformaldehyde, permeated with Triton $(0.5 \%, \mathrm{v} / \mathrm{v})$, blocked with BSA $(3 \%, w / v)$, and incubated with the anti-Akt antibodies (1:100) and anti-vimentin antibody (1:500) at $4^{\circ} \mathrm{C}$ for $12-16 \mathrm{~h}$. After extensive rinsing, the coverslips were incubated with secondary antibodies and stained with DAPI. The cell number on each coverslip was determined based on DAPI counting in nine random fields captured with a Leica TCS SP2 confocal microscope.

\section{Quantitative real-time PCR}

At least $10^{6}$ cells in each experimental group were collected for quantitative PCR analysis. Total RNA was extracted with TRIzol (Invitrogen) and reverse-transcribed to cDNA with the rtPCR kit. Quantitative real-time PCR was performed on a ViiA ${ }^{\mathrm{TM}} 7$ Real-Time PCR System (Applied Biosystems) to amplify GAPDH (housekeeping gene) and PDK1-4 with the SYBR qPCR kit. The primer sequences are shown in Supplementary Table 2. The amplification efficiency was evaluated by the standard curve method and was identified as $99.7 \%$ for GAPDH, 99.6\% for PDK1, 
99.7\% for $P D K 2,99.9 \%$ for $P D K 3$, and $97.0 \%$ for $P D K 4$. The mRNA levels were measured in three replicates and normalized to $G A P D H$ by the $-\triangle \triangle \mathrm{CT}$ method.

\section{siRNA transfection}

Passaged pOFs were seeded in 6-well plates to reach $80 \%$ confluence and transfected with RNAiMAX reagent according to the manufacturer's instruction. Briefly, after 6-h starvation, the pOFs were incubated in Opti-MEM (Gibco, Cat\# 31985088) containing RNAiMAX (vehicle group), RNAiMAX + scramble siRNA (Invitrogen, Cat\# 12935200; scramble group), or RNAiMAX + PDK2 siRNA (sense 5' $\rightarrow$ 3': GACCGAUGCUGUCAUCUAUTT; antisense 5' $\rightarrow$ 3': AUAGAUGACAGCAUCGGUCTT) for $24 \mathrm{~h}$. The transfected cells were harvested, replanted, or treated with other reagents for further experiments.

\section{Cytoplasmic protein extraction}

At least $10^{6}$ cells in each experimental group were collected for protein extraction. The cytoplasmic fractions were prepared according to the instruction of PARIS kit. Notably, the phosphatase inhibitor PhosSTOP ( $1 \times$ concentration, Roche) and the protease inhibitor PMSF ( $1 \mathrm{mM}$, Solarbio) reagents were added to the cell fractionation buffer in the PARIS kit.

\section{Capillary Western immunoassay (WES)}

Protein samples were quantified by a BCA protein assay (Beyotime) and immunodetected with the ProteinSimple WES ${ }^{\circledR}$ System according to the manufacturer's instruction. The relative amount of immunoreactive band was normalized to GAPDH in the same sample. Different loading concentration of protein samples were tested by a titration experiment, and the suitable concentration was determined as $0.5 \mu \mathrm{g} / \mu \mathrm{L}$ for PDK2 (1:50) and GAPDH (1:50) in total protein, $1.0 \mu \mathrm{g} / \mu \mathrm{L}$ for Akt (1:25) and GAPDH (1:25) in cytoplasmic protein, and $2.0 \mu \mathrm{g} / \mu \mathrm{L}$ for pAkt ${ }^{308}(1: 25)$ and GAPDH (1:25) in cytoplasmic protein.

\section{Statistics}

Statistical software was Statistical Product and Service Solutions (version 19.0). All outlier data were included in data analysis. Student's t-test was applied to compare two independent experimental groups. One-way ANOVA test was applied to compare three or more experimental groups from TAO pOFs or control pOFs. Two-way ANOVA test was applied to compare three or more experimental groups between the TAO and control OFs. If significant differences existed, Sidak test was applied for multiple comparisons. All data are expressed as the mean \pm s.D. A $P$ value less than 0.05 was considered statistically significant.

\section{Results}

\section{Clinical characteristics of recruited subjects}

Six TAO subjects and six control subjects were recruited in our study. The demographic characteristics including age (TAO: $54.0 \pm 4.7$, control: $43.5 \pm 5.5$ ), sex (three male and three female in both groups) and smoking status (TAO: two smokers, control: one smoker) are comparable between the TAO and control groups (Supplementary Table 1). The TAO subjects were at inactive stage with an average CAS of $0.8 \pm 0.3$ (range 0-2). The control subjects included three cases of orbital cavernous hemangioma, two cases of uveal melanoma, and one case of intermittent exotropia. For TAO and orbital tumor subjects, the extraocular muscle tissues were sampled at the muscle belly of the lateral rectus; for uveal melanoma and strabismus subjects, the extraocular muscle tissues were collected adjacent to the scleral insertion session of the medial rectus. All muscle tissues were processed for primary culture of pOFs immediately after collection, and passages 3-8 were used in the following experiments.

\section{DCA inhibits proliferation of TAO pOFs}

According to the CCK-8 assay, a high concentration of DCA (15-150 mM) dose-dependently suppressed the viability of TAO and control pOFs, while low concentration of DCA ( $<15 \mathrm{mM}$ ) showed no significant effects on cell viability (Fig. 1A). The effects of 0-150 mM DCA on proliferation were investigated by EdU and BrdU assays. Since incubation time significantly affected the proliferation results, the incubation time of EdU and BrdU was adjusted as 24 and $12 \mathrm{~h}$, respectively (Supplementary Fig. 1B, C and D). Both EdU and BrdU assays demonstrated that the TAO pOFs exhibited higher proliferation activity than the control pOFs (Fig. 1B, C, D and Supplementary Fig. 1B, C, D). DCA at 1--3 mM statistically suppressed TAO pOFs proliferation but showed no significant effects on control pOFs, while DCA at 5-15 $\mathrm{mM}$ inhibited proliferation of 
A
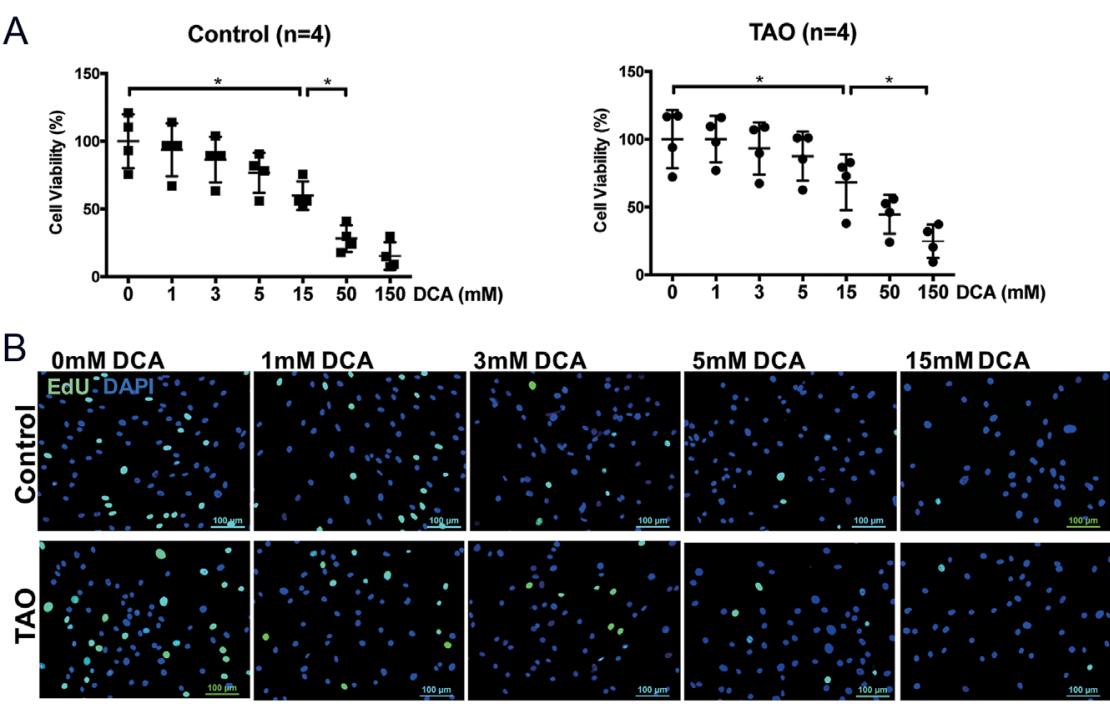

C EdU Immunofluorescence $(\mathrm{n}=4)$

$\mathrm{D}$

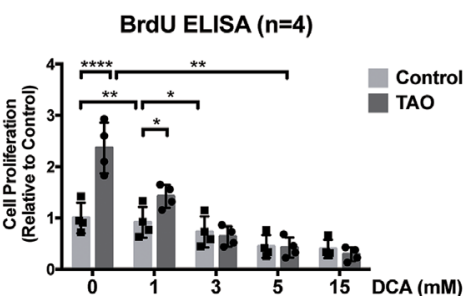

\begin{abstract}
Figure 1
DCA inhibits the proliferation of TAO pOFs. (A) The effects of different concentrations of DCA (0-150 $\mathrm{mM}$ ) on cell viability in the TAO and control pOFs. Each cell strain was repeatedly measured in six replicates, and cell viability was expressed as a percentage to the data in the $0 \mathrm{mM}$ group. ( $B$ and C) Comparison of cell proliferation based on EdU incorporation assay of the TAO and control pOFs treated with or without DCA (0-15 mM). The initial seeding density was $10^{4}$ cells/well in 24-well plates. The EdU-positive (green) nuclei (DAPI, blue) were counted in nine random fields of each cell strain. Scale bars, $100 \mu \mathrm{m}$. (D) Comparison of cell proliferation based on $\mathrm{BrdU}$ incorporation assay of the TAO and control pOFs treated with or without DCA (0-15 mM). The initial seeding density was 3500 cells/well in 96 -well plates. The absorbance value of each cell strain was calculated across six replicates and normalized to the data of control pOFs in the $0 \mathrm{mM}$ group. $\star P<0.05 ; * * P<0.01 ; * * * * P<0.000$;

$n$ indicates the number of cell strains in each experimental group.
\end{abstract}

both TAO and control pOFs (Fig. 1B and C). Notably, DCA at 50-150 mM resulted in large amounts of nonspecific binding in the EdU assay and significant increase of absorbance values in the BrdU assay, suggesting that 50-150 mM DCA is not suitable for cellular biological analysis (Supplementary Fig. $1 \mathrm{E}$ and F). The CCK-8 absorbance values were statistically lower in the TAO pOFs (Supplementary Fig. 1A), indicating that the TAO pOFs exhibited lower activity of dehydrogenases than the control pOFs. DCA is a specific inhibitor of glycolysis, and glycolytic cells are characterized with strong activity of proliferation and weak activity of pyruvate dehydrogenase (Stacpoole 2017). We, therefore, hypothesized that the glycolytic pathway may be enhanced in the TAO pOFs and the enhanced glycolysis, in turn, promotes proliferation.

\section{DCA suppresses the enhanced glycolysis in TAO pOFs}

Enhanced glycolytic pathway features increased lactate production and decreased oxygen consumption (Vander Heiden et al. 2009). The lactate production assay demonstrated a higher level of intracellular lactate in the TAO pOFs than the control pOFs, and DCA dose-dependently suppressed lactate production in the TAO pOFs (Fig. 2A). The oxygen consumption assay confirmed a lower oxygen consumption rate in the TAO pOFs than the control pOFs, and $1 \mathrm{mM}$ DCA statistically enhanced oxygen consumption in the TAO pOFs (Fig. 2B). Longer incubation time resulted in increased intracellular lactate concentration (Supplementary Fig. 2A), and therefore DCA treatment was fixed at $12 \mathrm{~h}$ for both lactate and oxygen measurement. After 12-h incubation, the cell numbers of TAO pOFs in the $5 \mathrm{mM}$ and $15 \mathrm{mM}$ DCA groups were statistically lower than the $0 \mathrm{mM}$ and $1 \mathrm{mM}$ DCA groups (Fig. 2C). Since oxygen consumption rate decreased as cell number reduced (Supplementary Fig. 2B), the uneven cell numbers may contribute to the paradoxical results that the oxygen consumption rate of TAO pOFs in the 5 and $15 \mathrm{mM}$ DCA groups were alower than the $1 \mathrm{mM}$ DCA group (Fig. 2B). The targets of DCA include four isotypes of PDK (Ki PDK2 < PDK1 $\approx$ PDK4 < PDK3) (Wu et al. 2019), and reverse-transcription PCR confirmed the existence of all four PDK mRNAs in both TAO and control pOFs (Supplementary Fig. 2C). Quantitative PCR revealed a higher level of PDK2 in the TAO pOFs than the control pOFs (Fig. 2D), suggesting that PDK2 overexpression may contribute to the enhanced glycolysis in the TAO pOFs. 
A Lactate Production $(n=4)$

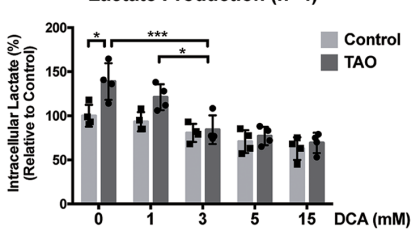

C

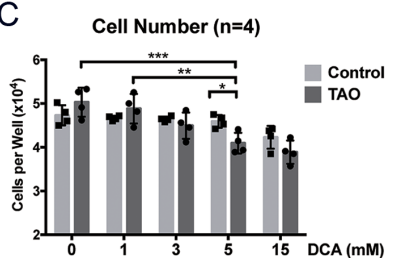

B
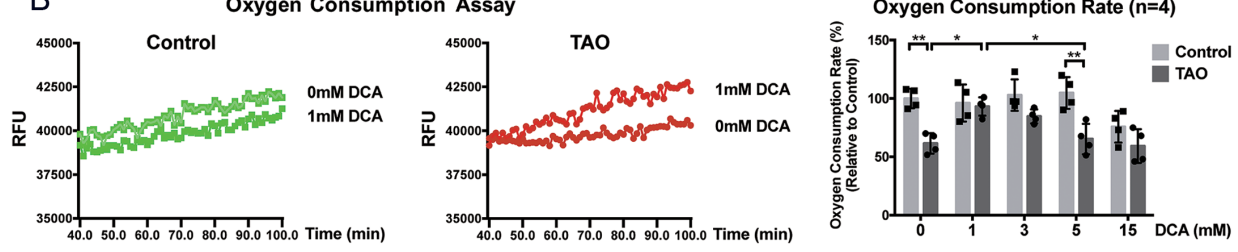

D

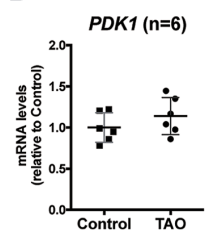

Quantitative PCR
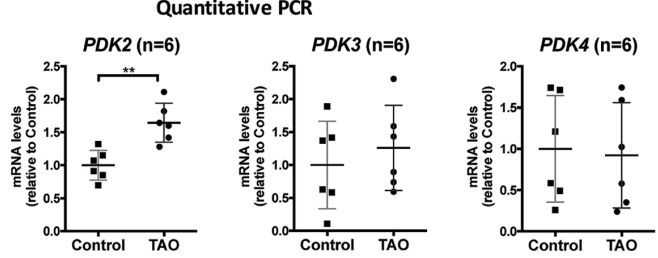

Figure 2

DCA suppresses the enhanced glycolysis in TAO pOFs. (A) Comparison of glycolysis based on intracellular lactate production of the TAO and control pOFs treated with or without DCA (0-15 mM). The lactate concentration of each sample was calculated across three replicates and expressed as a percentage to the data of control pOFs in the $0 \mathrm{mM}$ group. (B) Comparison of glycolysis based on the oxygen consumption rate of the TAO and control pOFs treated with or without DCA (0-15 mM). The line charts show representative signal profiles of one TAO (red) and one control (green) cell strain. The oxygen consumption rate was calculated based on linear slopes in three replicates and expressed as a percentage to the data of control pOFs in the $0 \mathrm{mM}$ group. (C) Corresponding cell numbers of each experimental group in (B). The cells were quantified by DAPI staining after measurement of oxygen consumption rate. (D) Comparison of $P D K$ mRNA levels in the TAO and control pOFs by quantitative PCR. Before RNA extraction, the cells were processed with 6-h starvation and 12 -h incubation in $1 \%(\mathrm{~V} / \mathrm{V}) \mathrm{FBS} . * P<0.05 ; * * P<0.01 ; * * * P<0.001 ; n$ indicates the number of cell strains in each experimental group.

\section{PDK2-enhanced glycolysis promotes proliferation of TAO pOFs}

The effects of PDK2 on glycolysis and proliferation were explored by siRNA transfection. Quantitative PCR confirmed that 24-h transfection with PDK2 siRNA (the siPDK2 group) significantly suppressed $P D K 2$ transcription in both TAO and control pOFs (Fig. 3A) and showed no significant effects on other PDK mRNA isotypes (Supplementary Fig. 3A). WES assay verified that the TAO pOFs expressed a higher level of PDK2 than the control pOFs, and PDK2 siRNA transfection for $24 \mathrm{~h}$ statistically decreased PDK2 protein levels in both TAO and control pOFs (Fig. 3B, C and Supplementary Fig. 3B, C, D). CCK-8 assay revealed that cell viability was not affected by siRNA transfection in both TAO and control groups (Fig. 1D). Congruent with the results in Supplementary Fig. 1A, the CCK-8 absorbance values of TAO pOFs were statistically lower than the control pOFs in the vehicle and scramble groups (Fig. 1D). Knockdown of $P D K 2$ resulted in decreased lactate production (Fig. 4A) and increased oxygen consumption (Fig. 4B) in the TAO pOFs but showed no significant effects in the control pOFs, providing evidence that the enhanced glycolysis in the TAO pOFs can be partially attributed to PDK2 overexpression. Knockdown of $P D K 2$ also exerted inhibitive effects on the proliferation of TAO pOFs, resulting in a significant decrease of EdU and BrdU incorporation in the siPDK2 group compared with the scramble group (Fig. 4C, D and E). The proliferation of control pOFs, however, was not affected by PDK2 siRNA transfection. Collectively, the above results establish that PDK2 overexpression enhances glycolysis and in turn promotes proliferation in the TAO pOFs.

\section{Cytoplasmic Akt participates in PDK2 signaling transduction in TAO pOFs}

Akt is a canonical signaling molecule to regulate proliferation, and cytoplasmic Akt has been recently identified as a candidate to regulate glycolysis (Chae et al. 2016). Immunofluorescence revealed Akt expression in both nucleus and cytoplasm, and the cytoplasmic Akt of the TAO pOFs was relatively lower in the siPDK2 group compared with the vehicle and scramble groups (Fig. 5A). The WES assays quantified Akt and its active form pAkt $^{308}$ in the cytoplasmic fractions of TAO and control pOFs (Fig. 5B, C and Supplementary Figs 4, 5). Based on data analysis, TAO pOFs expressed higher levels of Akt and pAkt ${ }^{308}$ than the control pOFs, and PDK 2 knockdown significantly suppressed Akt and pAkt ${ }^{308}$ expression in the TAO pOFs (Fig. 5D and E). These results collectively imply that cytoplasmic Akt may mediate the signaling transduction of PDK2.

A highly selective Akt inhibitor MK2206 was adapted to explore the role of Akt in PDK2 pathway. Quantitative PCR showed that PDK1-4 transcription was not affected 
A
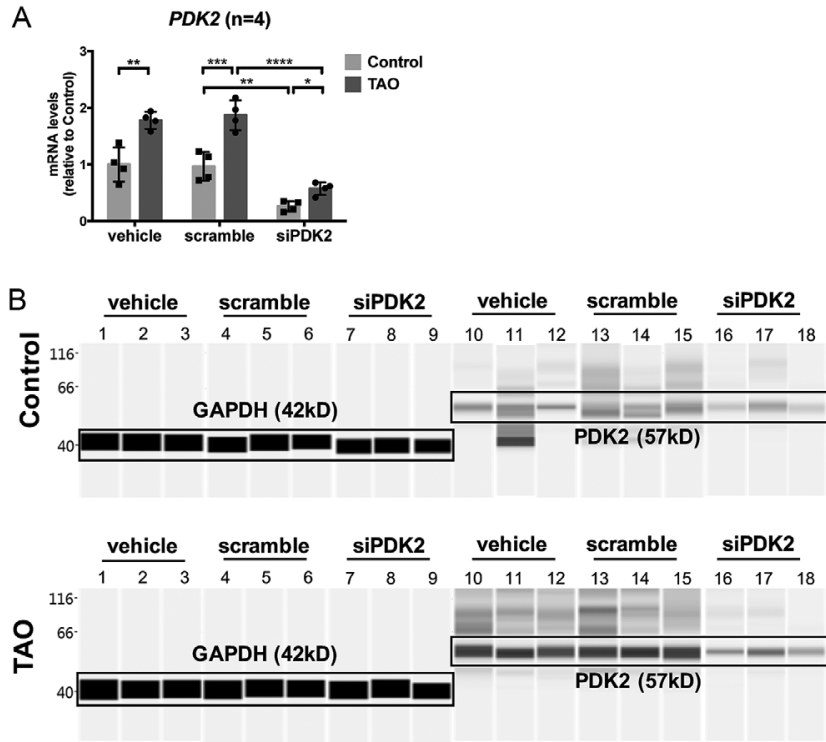

C

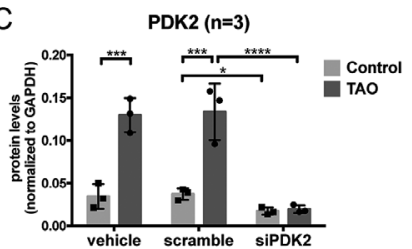

D

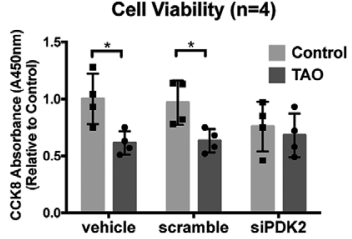

Figure 3

Verification of PDK2 knockdown in the TAO and control pOFs. (A) Verification of PDK2 knockdown by quantitative PCR in the TAO and control pOFs. The PDK2 mRNA levels were quantified in the vehicle (RNAiMAX reagent), scramble (scramble siRNA) and siPDK2 (PDK2 siRNA) groups and normalized to the data of control pOFs in the vehicle group. (B and C) Verification of PDK2 knockdown by WES chemiluminescence assay in the TAO and control pOFs. The PDK2 protein levels were quantified by the signal intensity in the virtual panels and normalized to GAPDH in each protein sample. Lanes 1, 4, 7, 10, 13, 16 represent one cel strain; lanes 2, 5, 8, 11, 14, 17 represent a second cell strain; lanes 3, 6, 9, $12,15,18$ represent a third cell strain. (D) The effects of PDK2 knockdown on cell viability in the TAO and control pOFs. Each cell strain was repeatedly measured in six replicates, and cell viability was expressed as a percentage to the CCK-8 absorbance values of control pOFs in the vehicle group. ${ }^{\star} P<0.05 ; * \star P<0.01 ; * \star * P<0.001 ; * \star * * P<0.000 ; n$ indicates the number of cell strains in each experimental group.

by MK2206 treatment (Fig. 6C and Supplementary Fig. 6A). The cell counting results confirmed that MK2206 significantly suppressed pOFs proliferation in both TAO and control groups (Fig. 6A and B), providing evidence that cytoplasmic Akt participates in PDK2 regulation of TAO pOFs proliferation. Regarding glycolysis, $3 \mu \mathrm{M}$ MK2206 statistically suppressed lactate production in the TAO pOFs but exerted no significant effects on control pOFs (Fig. 6D), indicating that Akt may play a role in promoting glycolysis in the TAO pOFs. The oxygen consumption rate of TAO pOFs slightly increased to 1.26-fold after incubation with $1 \mu \mathrm{M}$ MK2206, but data analysis detected no statistical significance (Fig. 6E). The incongruity between lactate and oxygen assays may result from the uneven cell numbers among different experimental groups after MK2206 treatment (Supplementary Fig. 6B). Taken together, cytoplasmic Akt in part mediates the promotive effects of PDK2 on glycolysis and proliferation in the TAO pOFs.

\section{Discussion}

Recently, the rapid advancement toward the understanding of oxygen homeostasis has yielded important insights into the pathogenesis of autoimmune disease. An inspiring concept has emerged that glycolysis plays a role in immune processes by promoting cell proliferation and strengthening antioxidant defense (Mills et al. 2017). With regard to TAO, there is increasing awareness of elevated oxidative stress within patient's orbit and peripheral blood (Korkmaz et al. 2015, Marique et al. 2015, Aass et al. 2016, Yuksel et al. 2019). It is reasonable to speculate that glycolysis is preferentially enhanced in TAO OFs so as to sustain prosperous proliferation and resist oxidative stress. Our observation, along with several previous studies, supported the speculation that enhanced glycolysis may be involved in TAO pathogenesis. For instance, orbital adipose-derived stem cells (OASCs) exhibited higher viability in the TAO group than the control group, and the increased viability is probably associated with a relatively higher expression of UCP1 which uncouples the oxidative phosphorylation and in turn, promotes glycolysis (Choi et al. 2018). High-throughput studies also disclosed potential candidates that may facilitate the glycolytic shift in TAO patients, such as downregulation of ALDH, downregulation of mitochondrial-encoded NADH and upregulation of AOC3 (Matheis et al. 2015, Tao et al. 2017, Kishazi et al. 2018). Furthermore, recent findings, albeit mostly circumstantial, supported the assumption that strong resistance to oxidative stress contributes to TAO development. According to in vitro studies, regardless of the $\mathrm{H}_{2} \mathrm{O}_{2}$ treatment to induce ROS production, the TAO OFs generated a lower amount of GSSG (product of ROS) and higher amount of GPX (enzyme to eliminate ROS) than the control OFs (Rotondo Dottore et al. 2017, $2018 a, b)$. Both histopathological study and proteomic analysis detected upregulation of antioxidant proteins in the tear and orbital tissues of TAO patients, suggesting stronger protection from oxidative damage (Marique et al. 2015, Aass et al. 2016). Given the lack of direct evidence, more investigation is needed before definitive conclusions can be drawn. Another ambiguous area concerns the proliferative capacity of TAO and control OFs. Some experts advocated that TAO OFs possessed a stronger 
A

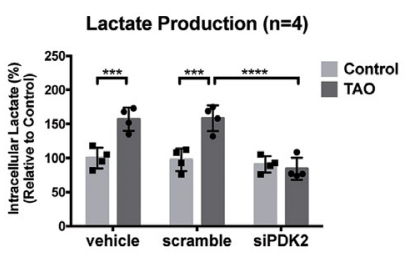

B

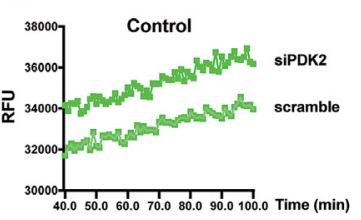

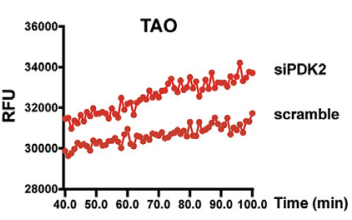

D

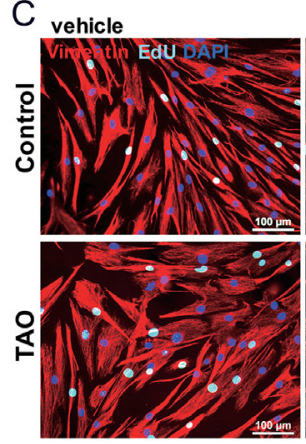

scramble

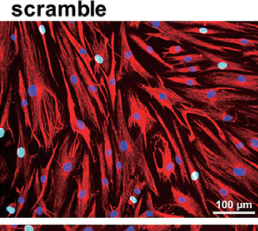

SIPDK2

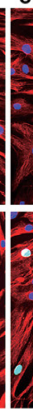

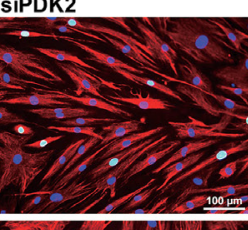

Ear

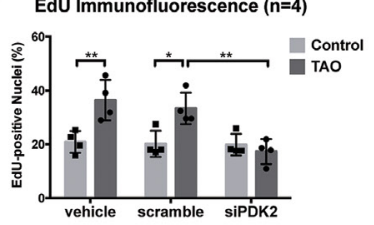

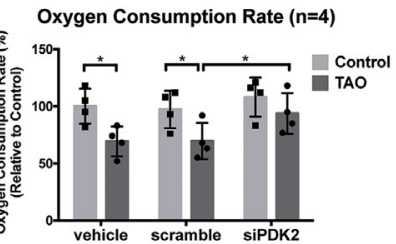

E

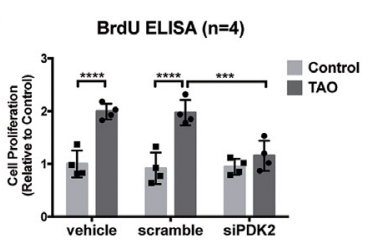

Figure 4

PDK2 knockdown inhibits glycolysis and proliferation in the TAO pOFs. (A) The effects of PDK2 knockdown on intracellular lactate production in the TAO and control pOFs. After transfection, approximately $2 \times 10^{6}$ cells in the vehicle, scramble and siPDK2 groups were harvested for lactate measurement. The lactate levels were calculated across three replicates and expressed as a percentage to the data of control pOFs in the vehicle group. (B) The effects of PDK2 knockdown on oxygen consumption rate in the TAO and control pOFs. The transfected cells were replanted to 96 -well plates at a density of $4 \times$ $10^{4}$ cells/well and processed with oxygen consumption assay. The line charts show representative signal profiles of one TAO (red) and one control (green) cell strain. The oxygen consumption rate was calculated across three replicates and expressed as a percentage to the data of control OFs in the vehicle group. (C and D) The effects of PDK2 knockdown on cell proliferation based on EdU incorporation assay in the TAO and control pOFs. Passaged cells were seeded at a density of $210^{4}$ cells/well in 24-well plates, processed with siRNA transfection, and incubated in 1\% (v/v) FBS for 48 h. The vimentin (red) staining demonstrates cell morphology, and the DAPI (blue) staining demonstrates nucleus. The EdU-positive (green) nuclei were counted in nine random fields of each cell strain to semi-quantify proliferation. Scale bars, $100 \mu \mathrm{m}$. (E) The effects of PDK2 knockdown on cell proliferation based on BrdU incorporation assay in the TAO and control pOFs. The transfected cells were replanted to 96 -well plates at a density of 4000 cells/well and incubated in $1 \%(\mathrm{v} / \mathrm{v})$ FBS for $48 \mathrm{~h}$. Each cell strain was measure in six replicates, and the absorbance values were normalized to the data of control pOFs in the vehicle group. $* P<0.05 ; * \star P<0.01 ; * \star \star P<0.001 ; * \star \star \star P<0.000 ; n$ indicates the number of cell strains in each experimental group.

capacity of proliferation than the control OFs, whereas others reported no significant differences between the TAO and control groups (Kau et al. 2016, Choi et al. 2018, Hikage et al. 2019, Woeller et al. 2019). Our study demonstrated a positive correlation between enhanced glycolysis and active proliferation in the TAO pOFs. We, therefore, assumed that the paradoxical conclusions may be attributed to a kinetic equilibrium between glycolysis and oxidative phosphorylation under different experimental conditions. Further study is necessary to investigate the oxygen homeostasis and its impact on cellular activities in the pathogenesis of TAO.

The progress in elucidating the function of PDK has invigorated novel therapeutic targets in various diseases. Inhibition of PDK with DCA blocks the glycolytic pathway and shows a beneficial effect in the treatment of diabetes, myocardial ischemia, lactic acidosis, and cancer (Stacpoole 2017). Cytoplasmic Akt was recently identified as a potent activator of PDK enzymes, and inhibition of Akt by MK2206 also enables a metabolic switch from cytoplasmic glycolysis to oxidative phosphorylation (Chae et al. 2016). In our study, the data of intracellular lactate concentration aligned with the above theories, while the data of oxygen consumption rate remains unexplained. The paradoxical results of oxygen consumption assay may be partially attributed to uneven cell density among different experiment groups. In addition, these results lead to the supposition that mitochondrial function may be impaired in the TAO pOFs. The influence of PDK activity on oxygen consumption is largely dependent on mitochondrial function. In cells with unimpaired mitochondria, inhibition of PDK activity restores mitochondrial glucose oxidation and subsequently promotes oxygen consumption (Denko 2008). In cells with defective mitochondrial activity, DCA treatment suppresses the energy flux by reducing oxygen consumption and consequently induces mitochondrial apoptosis (Martinez-Outschoorn et al. 2017). Growing evidence embraces the concept that mitochondrial defect may play a role in TAO pathogenesis. Electron microscopy studies directly observed mitochondrial abnormalities in the extraocular muscles obtained from TAO patients (Wall 
A

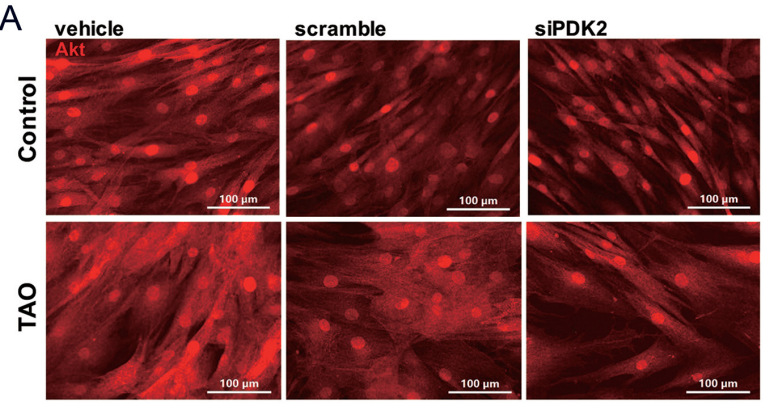

C

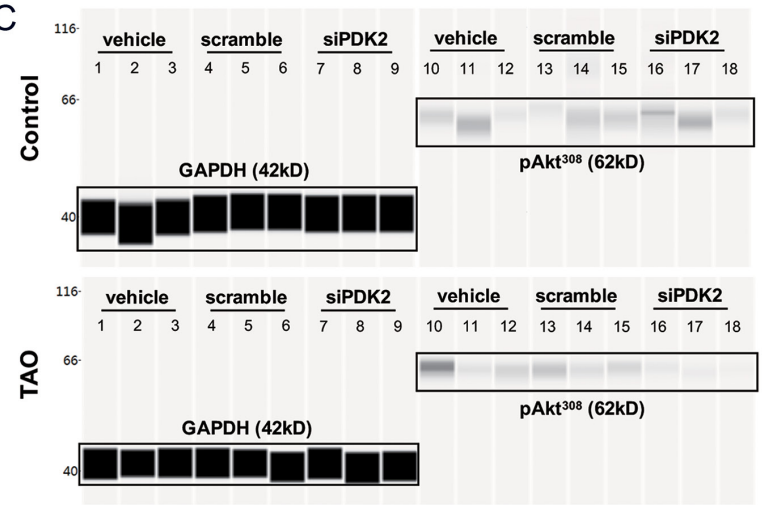

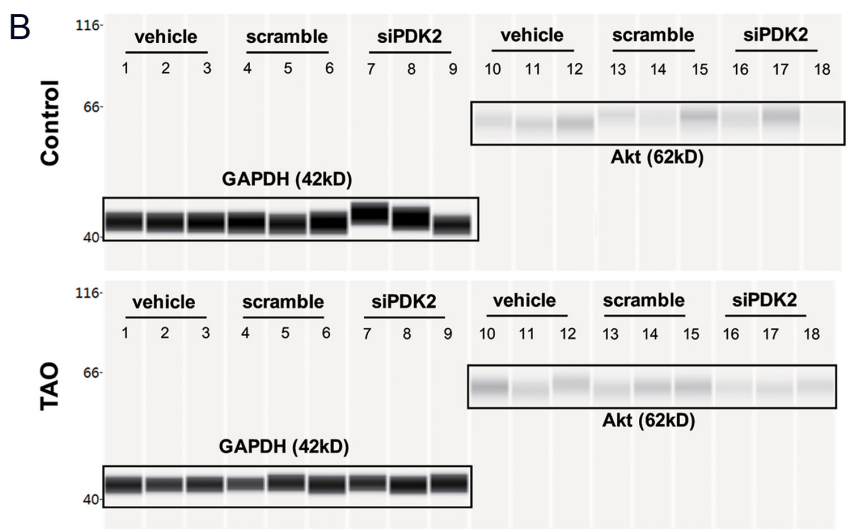

D

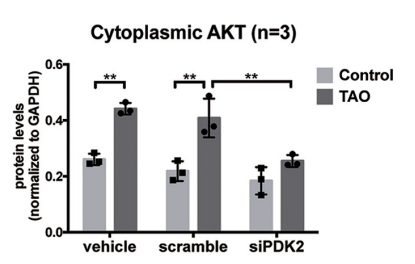

E
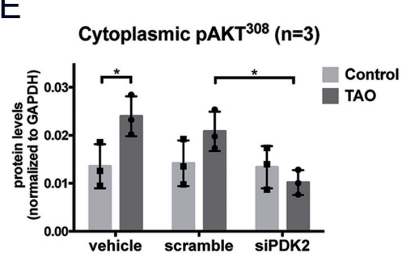

\section{Figure 5}

PDK2 knockdown suppresses cytoplasmic Akt activity in the TAO pOFs. (A) Akt immunofluorescence of the TAO and control pOFs in the vehicle (RNAiMAX reagent), scramble (scramble siRNA) and siPDK2 (PDK2 siRNA) groups. Scale bars, $100 \mu \mathrm{m}$. (B and C) The virtual panels of WES chemiluminescence assay to quantify Akt, pAkt ${ }^{308}$ and GAPDH in the cytoplasmic fractions of the TAO and control pOFs. The corresponding lanes of each immunostaining target (such as lanes 1 and 10) are results from a same protein sample. Lanes 1, 4, 7, 10, 13, 16 represent one cell strain; lanes 2, 5, 8, 11, 14, 17 represent a second cell strain; lanes 3, 6, 9, 12, 15, 18 represent a third cell strain. (D and E) Corresponding data analysis of (B) and (C). The relative amount of Akt and pAkt ${ }^{308}$ are quantified by the signal intensity and normalized to GAPDH in the same sample. $* P<0.05 ; * \star p<0.01 ; n$ indicates the number of cell strains in each experimental group.

et al. 2006, Tani et al. 2007). Autophagic vacuoles, which probably originated from defective mitochondria, were more abundant in the TAO OFs than the control OFs (Yoon et al. 2015). Several mitochondrial-specific genes, proteins and autoantibodies were dysregulated in the orbit and serum of TAO subjects compared with healthy control (Cheng et al. 2015, Matheis et al. 2015, Tao et al. 2017, Yuksel et al. 2019). Further investigation is required to determine whether mitochondrial impairment participates in TAO development.

Our data demonstrate a higher level of PDK2 mRNA in the TAO pOFs than the control pOFs. Further study is necessary to explore the mechanism of PDK2 overexpression in the TAO pOFs. According to previous findings, the transcription factor HIF $1 \alpha$ is a potential candidate. Stable expression of HIF1 $\alpha$ enables constitutive transactivation of target genes including PDKs and stimulates metabolic switch from oxidative phosphorylation to cytoplasmic glycolysis (Denko 2008). Previous research discovered an increase of HIF1 $\alpha$ in the
TAO OFs, and the HIF1 $\alpha$ level positively correlated with the clinical activity score of TAO patients (Görtz et al. 2016, Hai et al. 2020). In a recent study conducted with a three-dimensional (3D) culture system, however, the authors failed to detect the increased expression of HIF $1 \alpha$ but observed HIF $2 \alpha$ activation in the TAO group (Hikage et al. 2019). The incongruent findings may be attributed to the disparity of experimental conditions between 2D (cell layer) and 3D (cell organoid) culture settings. Thus, more effort should be directed in developing standardized approach to modeling TAO pathogenesis and exploring the regulatory network of oxygen homeostasis. Moreover, several pieces of evidence indicate that the cross talk between IGF-IR (insulin-like growth factor-I) and TSHR (thyrotropin receptor) may also participate in regulating oxidative stress in TAO orbit. In recent decades, a great progress in understanding TAO pathogenesis is the discovery of a physical and functional complex formed between the two receptor proteins. According to previous findings, TSHR downstream signaling is 
A 0uM MK2206

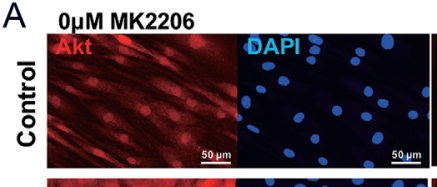

$1 \mu \mathrm{M}$ MK2206
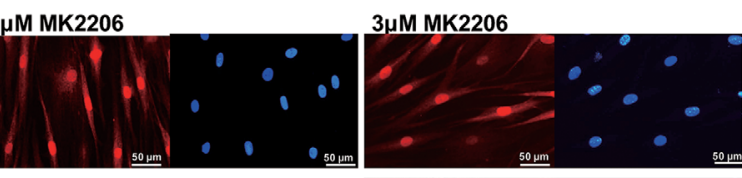

운
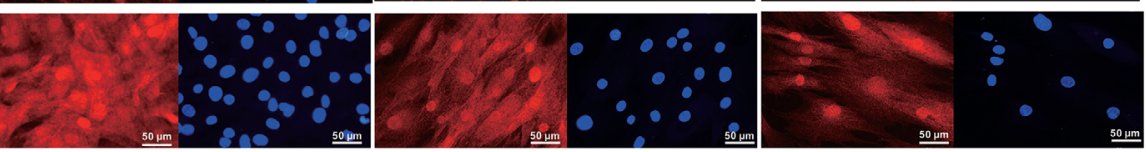

B

Cell Number $(n=3)$
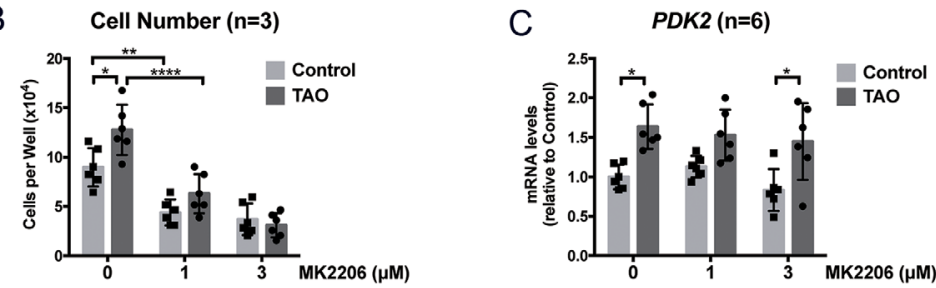

D Lactate Production ( $\mathrm{n}=3)$

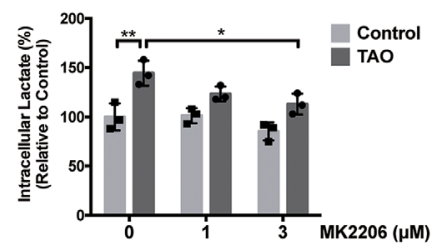

E
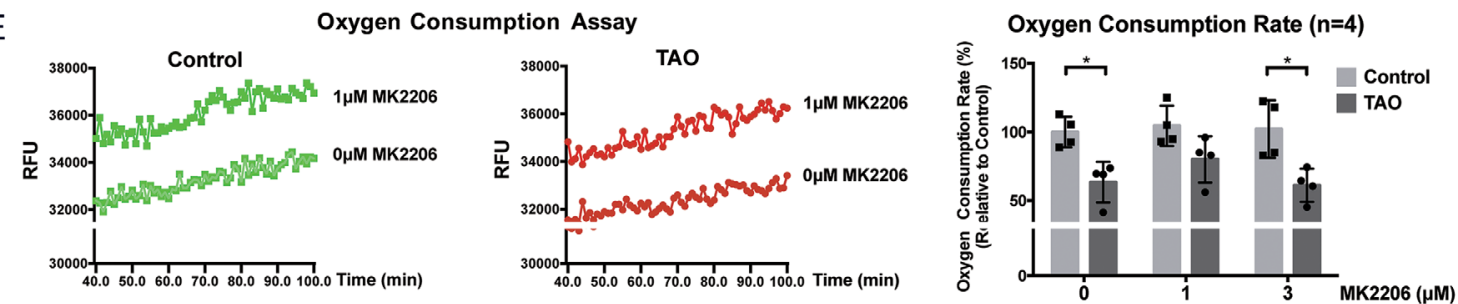

\section{Figure 6}

MK2206 inhibits proliferation and glycolysis in the TAO pOFs. (A) Akt (red) and DAPI (blue) immunofluorescence of the TAO and control pOFs treated with or without MK2206 (0-3 $\mu$ M). Cells were incubated with different concentrations of MK2206 in 1\% (v/v) FBS for $48 \mathrm{~h}$. Scale bars, $50 \mu \mathrm{m}$. (B) Corresponding cell numbers of each experimental group in (A). The cells were quantified by DAPI staining. The initial seeding density was $2 \times 10^{4}$ cells/well in 24 -well plates. (C) The effects of MK2206 (0-3 $\mu \mathrm{M}$ ) on PDK2 mRNA levels in the TAO and control pOFs. Cells were treated with or without MK2206 in $1 \%$ (v/v) FBS for $12 \mathrm{~h}$. The PDK2 mRNA levels were quantified by quantitative PCR and normalized to the data of control pOFs in the $0 \mu \mathrm{M}$ group. (D) The effects of MK2206 (0-3 $\mu \mathrm{M})$ on intracellular lactate production in the TAO and control pOFs. Cells were treated with or without MK2206 in $1 \%$ (v/v) FBS for $12 \mathrm{~h}$. The lactate levels were expressed as a percentage to the data of control pOFs in the $0 \mu \mathrm{M}$ group. (E) The effects of MK2206 (0-3 $\mu \mathrm{M})$ on the oxygen consumption rate of the TAO and control pOFs. The line charts show representative signal profiles of one TAO (red) and one control (green) cell strain. The oxygen consumption rate was calculated based on linear slopes in three replicates and expressed as a percentage to the data of control pOFs in the $0 \mu \mathrm{M}$ group. ${ }^{*} P<0.05 ; * \star P<0.01 ; * \star \star \star P<0.000 ; n$ indicates the number of cell strains in each experimental group.

strongly enhanced by IGF-IR activation and vice versa (Smith 2019). These findings collectively suggest that IGF-IR may play a critical role in mediating at least a subset of actions initiated through TSHR. IGF-IR is a membrane-spanning tyrosine kinase receptor that provokes Akt signaling and in turn, activates multiple biological processes including aerobic glycolysis (DiToro et al. 2020). Our study discovered that cytoplasmic Akt participated in promoting glycolysis and proliferation in the TAO group. These observations collectively provide the speculation that oxygen homeostasis may be part of the IGF-IR and TSHR signaling picture during TAO pathogenesis.

Pitfalls of our study stem from the control group chosen. Many factors, such as malignant tumor and tissue crowding, may interfere with oxygen homeostasis in extraocular muscles (Denko 2008, Vander Heiden et al. 2009). To minimize the effects of malignancy, the control group only recruited subjects with intrabulbar tumors (uveal melanoma), benign orbital tumors (cavernous hemangioma) and concomitant strabismus. To compromise the space-occupying effects, the muscle samples were collected from lateral recti to avoid orbital mass at supranasal quadrant. However, we anticipate more suitable control samples in future studies to investigate the regulation of aerobic glycolysis in extraocular muscle tissues. To conclude, PDK2 overexpression enhances glycolysis and promotes proliferation via Akt signaling transduction in the TAO pOFs, suggesting that PDK2 is a potential therapeutic target for TAO treatment. 


\section{Supplementary materials}

This is linked to the online version of the paper at https://doi.org/10.1530/ JME-20-0143.

\section{Declaration of interest}

The authors declare that there is no conflict of interest that could be perceived as prejudicing the impartiality of the research reported.

\section{Funding}

This work was supported by the National Natural Science Foundation of China (grant number 81970835, 81800867) and the Natural Science Foundation of Shanghai (grant number 20ZR1409500).

\section{Data availability}

The datasets generated and analyzed during the present study are available from the corresponding author on reasonable request.

\section{Author contribution statement}

$R M$ and $L G$ conceived and designed the experiments. $R M, L G$, and $H R$ performed the experiments. R M and L G analyzed the data. A H and J Q supervised the study. $R M$ and $L G$ wrote the paper. All authors revised the final version of the manuscript. J $Q$ is the principal investigator of this work and takes responsibility for the integrity of the data and the accuracy of the data analysis.

\section{Acknowledgements}

The authors gratefully thank Dr Yifei Yuan (Fudan Eye \& ENT Hospital), Dr Rui Zhang (Fudan Eye \& ENT Hospital) and Dr Yanqing Zhang (Fudan Eye \& ENT Hospital) for providing control orbital tissues.

\section{References}

Aass C, Norheim I, Eriksen EF, Børnick EC, Thorsby PM \& Pepaj M 2016 Comparative proteomic analysis of tear fluid in Graves' disease with and without orbitopathy. Clinical Endocrinology 85 805-812. (https:// doi.org/10.1111/cen.13122)

Byun JW, Hwang S, Kang CW, Kim JH, Chae MK, Yoon JS \& Lee EJ 2016 Therapeutic effect of protocatechuic aldehyde in an in vitro model of Graves' orbitopathy. Investigative Ophthalmology and Visual Science 57 4055-4062. (https://doi.org/10.1167/iovs.15-19037)

Chae YC, Vaira V, Caino MC, Tang HY, Seo JH, Kossenkov AV, Ottobrini L, Martelli C, Lucignani G, Bertolini I, et al. 2016 Mitochondrial Akt regulation of hypoxic tumor reprogramming. Cancer Cell 30 257-272. (https://doi.org/10.1016/j. ccell.2016.07.004)

Cheng KC, Hung CT, Cheng KY, Chen KJ, Wu WC, Suen JL, Wu YJ \& Chang CH 2015 Proteomic surveillance of putative new autoantigens in thyroid orbitopathy. British Journal of Ophthalmology 99 1571-1576. (https://doi.org/10.1136/bjophthalmol-2015-306634)

Choi CJ, Tao W, Doddapaneni R, Acosta-Torres Z, Blessing NW, Lee BW, Pelaez D \& Wester ST 2018 The effect of prostaglandin analogue bimatoprost on thyroid-associated orbitopathy. Investigative Ophthalmology and Visual Science 59 5912-5923. (https://doi. org/10.1167/iovs.18-25134)
Denko NC 2008 Hypoxia, HIF1 and glucose metabolism in the solid tumour. Nature Reviews: Cancer 8 705-713. (https://doi.org/10.1038/ $\operatorname{nrc2468)}$

DiToro D, Harbour SN, Bando JK, Benavides G, Witte S, Laufer VA, Moseley C, Singer JR, Frey B, Turner H, et al. 2020 Insulin-like growth factors are key regulators of $\mathrm{T}$ helper 17 regulatory $\mathrm{t}$ cell balance in autoimmunity. Immunity 52 650-667.

Douglas RS, Kahaly GJ, Patel A, Sile S, Thompson EHZ, Perdok R, Fleming JC, Fowler BT, Marcocci C, Marinò M, et al. 2020 Teprotumumab for the treatment of active thyroid eye disease. New England Journal of Medicine 382 341-352. (https://doi.org/10.1056/ NEJMoa1910434)

Görtz GE, Horstmann M, Aniol B, Reyes BD, Fandrey J, Eckstein A \& Berchner-Pfannschmidt U 2016 Hypoxia-dependent HIF-1 activation impacts on tissue remodeling in Graves' ophthalmopathyimplications for smoking. Journal of Clinical Endocrinology and Metabolism 101 4834-4842. (https://doi.org/10.1210/jc.2016-1279)

Hai YP, Lee ACH, Frommer L, Diana T \& Kahaly GJ 2020 Immunohistochemical analysis of human orbital tissue in Graves' orbitopathy. Journal of Endocrinological Investigation 43 123-137. (https://doi.org/10.1007/s40618-019-01116-4)

Hikage F, Atkins S, Kahana A, Smith TJ \&Chun TH 2019 HIF2A-LOX pathway promotes fibrotic tissue remodeling in thyroid-associated orbitopathy. Endocrinology 160 20-35. (https://doi.org/10.1210/ en.2018-00272)

Kau HC, Wu SB, Tsai CC, Liu CJ \& Wei YH 2016 Cigarette smoke extract-induced oxidative stress and fibrosis-related genes expression in orbital fibroblasts from patients with Graves' ophthalmopathy. Oxidative Medicine and Cellular Longevity 2016 4676289. (https://doi. org/10.1155/2016/4676289)

Kishazi E, Dor M, Eperon S, Oberic A, Hamedani M \& Turck N 2018 Thyroid-associated orbitopathy and tears: a proteomics study. Journal of Proteomics 170 110-116. (https://doi.org/10.1016/j. jprot.2017.09.001)

Ko J, Kim JY, Lee EJ \& Yoon JS 2018 Inhibitory effect of idelalisib, a selective phosphatidylinositol 3-kinase $\delta$ inhibitor, on adipogenesis in an in vitro model of Graves' orbitopathy. Investigative Ophthalmology and Visual Science 59 4477-4485. (https://doi. org/10.1167/iovs.18-24509)

Korkmaz H, Tabur S, Ozkaya M, Oguz E, Aksoy N \& Akarsu E 2015 Serum prolidase levels in Graves' disease without ophthalmopathy and its association with oxidative status. Journal of Endocrinological Investigation 38 1167-1173. (https://doi.org/10.1007/s40618-0150278-7)

Lee JS, Kim J, Lee EJ \& Yoon JS 2019 Therapeutic effect of curcumin, a plant polyphenol extracted from curcuma longae, in fibroblasts from patients with Graves' orbitopathy. Investigative Ophthalmology and Visual Science 60 4129-4140. (https://doi.org/10.1167/iovs.19-27376)

Ma R, Li Q, Wang Z, Yuan Y, Gan L \& Qian J 2018 Modulation of hyaluronan polymer size regulates proliferation of perimysial fibroblasts in thyroid eye disease. Biochemical and Biophysical Research Communications 496 1376-1381. (https://doi.org/10.1016/j. bbrc.2018.02.037)

Ma R, Ren H, Xu B, Cheng Y, Gan L, Zhang R, Wu J \& Qian J 2019 PH20 inhibits TGF $\beta 1$-induced differentiation of perimysial orbital fibroblasts via hyaluronan-CD44 pathway in thyroid-associated ophthalmopathy. Investigative Ophthalmology and Visual Science 60 1431-1441. (https://doi.org/10.1167/iovs.18-26268)

Marique L, Senou M, Craps J, Delaigle A, Van Regemorter E, Wérion A, Van Regemorter V, Mourad M, Nyssen-Behets C, Lengelé B, et al. 2015 Oxidative stress and upregulation of antioxidant proteins, including adiponectin, in extraocular muscular cells, orbital adipocytes, and thyrocytes in Graves' disease associated with orbitopathy. Thyroid $\mathbf{2 5}$ 1033-1042. (https://doi.org/10.1089/thy.2015.0087)

Martinez-Outschoorn UE, Peiris-Pagés M, Pestell RG, Sotgia F \& Lisanti MP 2017 Cancer metabolism: a therapeutic perspective. 
Nature Reviews: Clinical Oncology 14 11-31. (https://doi.org/10.1038/ nrclinonc.2016.60)

Matheis N, Lantz M, Grus FH, Ponto KA, Wolters D, Brorson H, Planck T, Shahida B, Pitz S, Pfeiffer N, et al. 2015 Proteomics of orbital tissue in thyroid-associated orbitopathy. Journal of Clinical Endocrinology and Metabolism 100 E1523-E1530. (https://doi. org/10.1210/jc.2015-2976)

Mills EL, Kelly B \& O'Neill LAJ 2017 Mitochondria are the powerhouses of immunity. Nature Immunology 18 488-498. (https://doi. org/10.1038/ni.3704)

Rotondo Dottore G, Leo M, Casini G, Latrofa F, Cestari L, SellariFranceschini S, Nardi M, Vitti P, Marcocci C \& Marinò M 2017 Antioxidant actions of selenium in orbital fibroblasts: a basis for the effects of selenium in Graves' orbitopathy. Thyroid 27 271-278. (https://doi.org/10.1089/thy.2016.0397)

Rotondo Dottore G, Ionni I, Menconi F, Casini G, Sellari-Franceschini S, Nardi M, Vitti P, Marcocci C \& Marinò M 2018a Action of three bioavailable antioxidants in orbital fibroblasts from patients with Graves' orbitopathy (GO): a new frontier for GO treatment? Journal of Endocrinological Investigation 41 193-201. (https://doi.org/10.1007/ s40618-017-0718-7)

Rotondo Dottore G, Ionni I, Menconi F, Casini G, Sellari-Franceschini S, Nardi M, Vitti P, Marcocci C \& Marinò M 2018b Antioxidant effects of $\beta$-carotene, but not of retinol and vitamin $\mathrm{E}$, in orbital fibroblasts from patients with Graves' orbitopathy (GO). Journal of Endocrinological Investigation 41 815-820. (https://doi.org/10.1007/ s40618-017-0809-5)

Smith TJ 2019 Challenges in orphan drug development: identification of effective therapy for thyroid-associated ophthalmopathy. Annual Review of Pharmacology and Toxicology 59 129-148. (https://doi. org/10.1146/annurev-pharmtox-010617-052509)

Smith TJ \& Janssen JAMJL 2019 Insulin-like growth factor-I receptor and thyroid-associated ophthalmopathy. Endocrine Reviews 40 236-267. (https://doi.org/10.1210/er.2018-00066)

Stacpoole PW 2017 Therapeutic targeting of the pyruvate dehydrogenase complex/pyruvate dehydrogenase kinase (PDC/PDK) axis in cancer. Journal of the National Cancer Institute 109 djx071. (https://doi. org/10.1093/jnci/djx071)

Tani J, Gopinath B, Nguyen B \& Wall JR 2007 Extraocular muscle autoimmunity and orbital fat inflammation in thyroid-associated ophthalmopathy. Expert Review of Clinical Immunology 3 299-311. (https://doi.org/10.1586/1744666X.3.3.299)

Tao W, Ayala-Haedo JA, Field MG, Pelaez D \& Wester ST 2017 RNAsequencing gene expression profiling of orbital adipose-derived stem cell population implicate HOX genes and WNT signaling dysregulation in the pathogenesis of thyroid-associated orbitopathy. Investigative Ophthalmology and Visual Science 58 6146-6158. (https:// doi.org/10.1167/iovs.17-22237)

Taylor PN, Zhang L, Lee RWJ, Muller I, Ezra DG, Dayan CM, Kahaly GJ \& Ludgate M 2020 New insights into the pathogenesis and nonsurgical management of Graves orbitopathy. Nature Reviews: Endocrinology 16 104-116. (https://doi.org/10.1038/s41574019-0305-4)

Vander Heiden MG, Cantley LC \& Thompson CB 2009 Understanding the Warburg effect: the metabolic requirements of cell proliferation. Science 324 1029-1033. (https://doi.org/10.1126/science.1160809)

Wall JR, Stachura I \& Kennerdell JH 2006 Mitochondrial abnormalities in eye muscle fiber from three cases of thyroid-associated ophthalmopathy. Thyroid 16 1181-1183. (https://doi.org/10.1089/ thy.2006.16.1181)

Wiersinga W, Žarković M, Bartalena L, Donati S, Perros P, Okosieme O, Morris D, Fichter N, Lareida J, von Arx G, et al. 2018 Predictive score for the development or progression of Graves' orbitopathy in patients with newly diagnosed Graves' hyperthyroidism. European Journal of Endocrinology 178 635-643. (https://doi.org/10.1530/EJE18-0039)

Woeller CF, Roztocil E, Hammond C \& Feldon SE 2019 TSHR signaling stimulates proliferation through PI3K/Akt and induction of miR146a and miR-155 in thyroid eye disease orbital fibroblasts. Investigative Ophthalmology and Visual Science 60 4336-4345. (https:// doi.org/10.1167/iovs.19-27865)

Wu DM, Wang YJ, Fan SH, Zhang ZF, Shan Q, Lu J, Chen GQ \& Zheng YL 2019 High-throughput screening of novel pyruvate dehydrogenase kinases inhibitors and biological evaluation of their in vitro and in vivo antiproliferative activity. European Journal of Medicinal Chemistry 164 252-262. (https://doi.org/10.1016/j. ejmech.2018.12.051)

Yoon JS, Lee HJ, Chae MK \& Lee EJ 2015 Autophagy is involved in the initiation and progression of Graves' orbitopathy. Thyroid $\mathbf{2 5}$ 445-454. (https://doi.org/10.1089/thy.2014.0300)

Yuksel N, Tanriverdi B, Ipteç B \& Erel O 2019 Thiol-disulfide homeostasis as an oxidative stress marker in patients with Graves' ophthalmopathy. Orbit 38 370-375. (https://doi.org/10.1080/016768 30.2018.1553191)

Yuksel N, Yaman D, Tugce Pasaoglu O \& Pasaoglu H 2020 The effect of smoking on mitochondrial biogenesis in patients with graves ophthalmopathy. Ophthalmic Plastic and Reconstructive Surgery 36 172-177. (https://doi.org/10.1097/IOP.0000000000001514)

Received in final form 7 September 2020

Accepted 21 September 2020

Accepted Manuscript published online 21 September 2020 (c) 2020 Society for Endocrinology Published by Bioscientifica Ltd. Printed in Great Britain 